\title{
SIMULATION OF CELLULAR STRUCTURES WITH A COUPLED FEM-FCM APPROACH BASED ON CT DATA
}

\author{
UlRich GabBert and Mathias WÜRKneR \\ Otto von Guericke University of Magdeburg \\ Universitaetsplatz 2, 39114 Magdeburg, Germany \\ ulrich.gabbert@ovgu.de, mathias.wuerkner@ovgu.de
}

[Received: October 30, 2020; Accepted: December 11, 2020]

Dedicated to Professor Barna Szabó on the occasion of his eighty-fifth birthday

\begin{abstract}
The application of cellular structural materials provide new light-weight capabilities in many engineering fields. But the microstructure significantly influences the strength, the fatigue and fracture behavior as well as the life span of a structure made from cellular materials. The current paper illustrates the general idea how to take into account the cellular microstructure in the stress and strain analysis. The detailed geometry, including all discontinuities in the microstructure is available, for instance from measurements provided by the computed tomography $(\mathrm{CT})$. The proposed simulation methodology is a combination of the finite element method (FEM) and the finite cell method (FCM). The FCM approach is applied in regions where discontinuities occur, avoiding a body-fitted mesh. As basis of the FEM-FCM coupling the commercial FEA package Abaqus is used. The theoretical background and the overall simulation workflow along with specific implementation details are discussed. Finally, academic benchmark problems are used to verify the developed coupling method.
\end{abstract}

Mathematical Subject Classification: 65L05, 76G25

Keywords: Finite element method (FEM), finite cell method (FCM) , computed tomography (CT), cellular structures, coupling of the FEM with the FCM

\section{INTRODUCTION}

The application of ultra-light-weight constructions contributes to a reduction of natural resources in many fields of engineering. There are several types of light-weight materials available, such as cellular materials made of aluminium or plastic foam, sandwich materials with a core layer from honeycomb, hollow spheres or foam, etc. The increasing application of additive manufacturing technologies also allows the production of complex light-weight components with a special designed porosity, which can also be seen as a cellular structure as well. The general application of cellular materials provides new design capabilities in several engineering fields, such as in automotive and aerospace industries, electro engineering, wind power industries, machine and plant engineering, container constructions, etc. Besides the application of specially designed cellular materials there are other lightweight structures with great advantages, particularly in mass production, e.g. aluminium die cast parts. 
Components produced with the die cast technology and with additive manufacturing have local inhomogeneities, such as pores in die cast parts and voids in additive produced parts, which are unavoidable. Such local porosities can be recognised with help of computed tomography (CT).

The desired and also the undesired inhomogeneities in the microstructure have to be taken into account in the simulation, because they significantly influence the strength, the fatigue and fracture behavior and, consequently, also the life span of a structure.

The main approach to analyse engineering structures is the application of the finite element method [1 3]. The typical method to take into account the microstructure in a global $\mathrm{FE}$ analysis is a homogenisation of the microstructure. Homogenisation means, that with help of different methods the microstructure is smeared, resulting in a homogenised reference material with the same global behavior as the original material with the microstructure. One standard homogenisation approach is based on the Hill theory [4]. There are several homogenisation methods available, such as the Eshelby-based methods, the Mori-Tanaka method, the generalised self-consistent method, the asymptotic homogenisation method, the representative volume element approach (RVE) etc.; for an overview see [5] 9]. Of course, the local behaviour, e.g. the local stress-strain state, is lost when applying homogenisation methods. In FEM the microstructure can be taken into account, e.g. by a substructure approach [10] or by a multilevel finite element method (FE2) approach 11,12 . In both cases the microstructure has to be meshed in some detail, which results in an increase of the computational effort.

One important problem is the generation of a high quality body-fitted mesh as a basis for the FEM. This pre-processing step calls for an experienced designer and can account for up to $80 \%$ of the overall analysis time [13. In order to capture the microstructure a refined finite element mesh is needed to ensure an accurate approximation of the geometry. Most cellular materials have a randomly sized and distributed microstructure. To simplify the meshing procedure a reference microstructure can be used, which is regular and statistically equivalent to the original microstructure. In this case the finite element mesh can be generated automatically. The disadvantage is the loss of the irregularity of the microstructure. Alternatively, the micro-structure can be obtained from computed tomography (CT) images [14].

The most promising approach to avoid a finite element mesh fitting the geometry of the cellular microstructure is the application of the finite cell method (FCM). The FCM can also simply process CT data 15,16 . The method can be automated straightforwardly and thus reduces the required input data. In [17] we have successfully implemented the FCM in order to analyse the propagation of ultrasonic waves in heterogeneous structures. The application of higher order finite elements increases the accuracy and reduces the required computational effort [18. In this case a rough finite element approximation results in very accurate solutions even if a fine microstructure has to be analysed. A first in-house code based on the higher order FCM has been developed by S. Duczek 19, 20. 
The FCM has also great advantages for structures with a cellular microstructure, which is often limited to small regions, such as in die cast parts 21]. The remaining parts of a structure can be well approximated by the classical FEM. Consequently, a combination of the FEM and the FCM, where the FCM is only applied in regions with local heterogeneities, could be of great advantage. The regions with a local microstructure known from CT scans can be simply meshed with hexagonal cells, avoiding complicated body adapted mesh generation.

Most of the FE simulations in industry are performed with commercial FEA tools, such as Abaqus, Ansy, Nastran and others. Therefore, it would be of a great industrial interest if the FCM approach would be a part of commercial FEA software $[22$. In the following a concept of an overall workflow to combine the FCM with the commercial FE software Abaqus is presented and tested, where also specific implementation details and application problems are discussed. The paper is organized as follows. In Section 2 the fundamental principles of the three-dimensional finite cell method are briefly recalled. This also includes the main differences to the classical finite element method. In Section 3 the coupling concept based on the commercial analysis tool Abaqus is described. In Section 4 some information and trouble shooting according to the STL data derived from CT measurements is given. In Section 5 the developed coupling procedure is tested by analyzing an academic test example, which demonstrates the capability of the developed simulation concept. The paper finishes with conclusions in Section 6 .

\section{The Finite CELL METHOD}

In the following we briefly summarize the basics of the FCM, assuming that the FEM is well known. The FCM slightly differs from the FEM, and, consequently, we start with the typical basics from the FEM. We assume a linear elastic static boundary value problem. The solution $\mathbf{u}$ in a region $\Omega$ is equivalent to the solution of the variational form

$$
B(\mathbf{u}, \mathbf{v})=F(\mathbf{v}), \quad \forall \mathbf{v} \in V .
$$

This is the weak form of the equilibrium conditions of the problem. Here $\mathbf{u}$ is the displacement vector and $\mathbf{v}$ represents the vector of arbitrary test functions in the space $V$ of admissible functions. The bilinear form $B$ and the linear form $F$ are given as follows

$$
\begin{gathered}
B(\mathbf{u}, \mathbf{v})=\int_{\Omega}[\mathbf{L v}]^{T} \mathbf{C}[\mathbf{L u}] \mathrm{d} \Omega, \\
F(\mathbf{v})=\int_{\Omega} \mathbf{v}^{T} \overline{\mathbf{f}} \mathrm{d} \Omega+\int_{\Gamma_{N}} \mathbf{v}^{T} \overline{\mathbf{t}} \mathrm{d} \Gamma .
\end{gathered}
$$

Here $\mathbf{L}$ denotes the linear strain-displacement operator, $\mathbf{C}$ stands for the Hook elasticity matrix, $\mathbf{f}$ denotes the vector of body forces and $\mathbf{t}$ is the traction vector. A bar over a variable signifies a prescribed value. The prescribed tractions are defined on the Neumann boundary $\Gamma_{N}$ as

$$
\boldsymbol{\sigma} \mathbf{n}=\overline{\mathbf{t}} \quad \text { on } \Gamma_{\mathrm{N}}
$$


where here $\boldsymbol{\sigma}$ denotes the stress tensor and $\mathbf{n}$ constitutes the outward normal vector of unit length. Furthermore the displacements are prescribed on the Dirichlet boundary $\Gamma_{D}$, resulting in

$$
\mathbf{u}=\overline{\mathbf{u}} \quad \text { on } \Gamma_{\mathrm{D}}
$$

Equations (2.1)-2.5) are the general basis of FEM [3]. In order to solve a problem with the help of FEM a mesh of finite elements is required which approximately coincides with the geometry of the structural region of interest $\Omega$. But in FCM the mesh in general must not fit the structural geometry. The physical domain $\Omega$ is extended by a fictitious domain $\Omega_{\text {fic }}$. The union of these two domains forms the extended domain $\Omega_{\mathrm{ex}}-$ see Figure 1 .

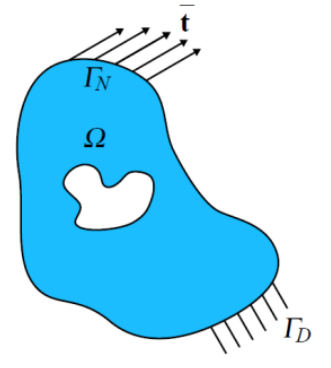

(a) Physical domain $\Omega$

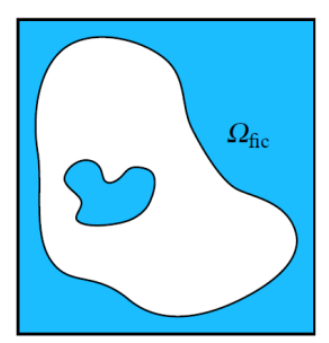

(b) Fictitious domain $\Omega_{\text {fic }}$

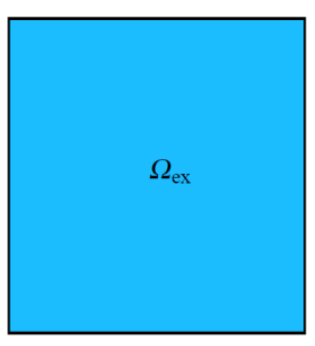

(c) Extended domain $\Omega_{\mathrm{ex}}$

Figure 1. Fictitious domain approach

Instead of equation (2.1) the weak form is now solved over the extended region

$$
B_{\text {ex }}(\mathbf{u}, \mathbf{v})=F_{\text {ex }}(\mathbf{v}), \quad \forall \mathbf{v} \in V .
$$

The main advantage of the fictitious domain approach is that the extended domain is of a much simpler geometry and can, therefore, be simply meshed by regular nondistorted finite elements. Quadrilateral and hexagonal elements and triangular and tetrahedral elements can be used for 2D and 3D problems, respectively. During the analysis it is imported to distinguish between normal (not cut) finite elements and elements cut by the physical boundary. This differentiation is controlled by the socalled indicator function $\alpha$ as

$$
\alpha(\mathbf{x})=\left\{\begin{array}{cl}
1 & \forall \mathbf{x} \in \Omega \\
a_{0}=10^{-q} & \forall \mathbf{x} \in \Omega_{e x} \backslash \Omega
\end{array}\right.
$$

If $\mathbf{x}$ is in the fictitious region, the indicator function can be taken as zero. In order to avoid numerical problems a small value $a_{0}$ is used instead of zero. The exponent $q$ is typically taken in the range from 4 to 15 , depending on the material properties [23. With the value $\alpha$ equations $(2.2)$ and $(2.3)$ are modified as

$$
\begin{gathered}
B_{\mathrm{ex}}(\mathbf{u}, \mathbf{v})=\int_{\Omega_{\mathrm{ex}}}[\mathbf{L} \mathbf{v}]^{T} \alpha \mathbf{C}[\mathbf{L u}] \mathrm{d} \Omega, \\
F_{\mathrm{ex}}(\mathbf{v})=\int_{\Omega_{\mathrm{ex}}} \mathbf{v}^{T} \alpha \overline{\mathbf{f}} \mathrm{d} \Omega+\int_{\Gamma_{N}} \mathbf{v}^{T} \overline{\mathbf{t}} \mathrm{d} \Gamma .
\end{gathered}
$$


The indicator function $\alpha$ allows distinguishing between points that are located in $\Omega$ or in $\Omega_{\text {fic }}$, respectively. In Figure 2 a simple rectangular mesh is shown. This mesh is not aligned to the physical boundary of the structure, marked with a dotted red line.

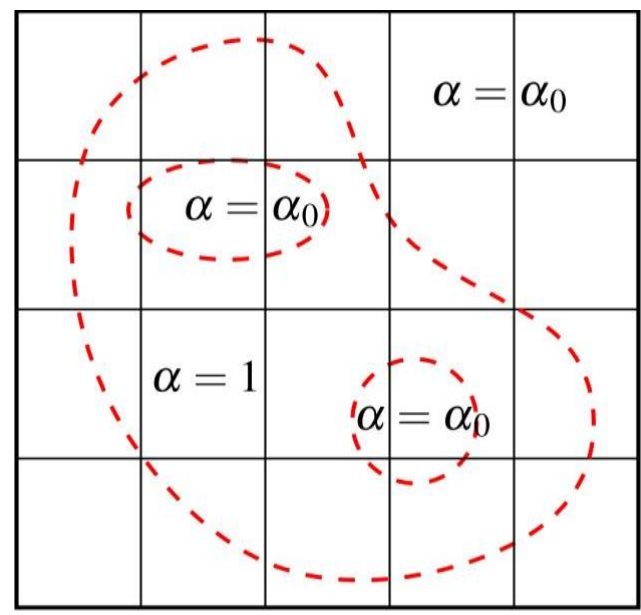

Figure 2. Finite cell discretization

The problem now arises that the numerical integration of the element matrices of the cut elements has to be performed over discontinuous integrands. The usual applied Gaussian integration of the element matrices is in such cases not accurate enough. Therefore, an adaptive Gaussian integration rule is recommended [15], which can be applied automatically in 2D as well as in 3D finite elements without any extra manual input. For this purpose a space tree partitioning (e.g. a quadtree in 2D and an octree in 3D) of the integration domain is executed, and in each cut element of the extended region $\Omega_{\text {ex }}$ a Gaussian integration rule is used 11. The partitioning is carried out as long as the solution of the integral is sufficiently accurately approximated. Figure 3 shows such a partitioning of an element in integration subdomains. Alternatively to the space tree subdivision in [24, an efficient integration scheme based on the divergence theorem (Gauss-Ostrogradsky theorem) can be applied, which reduces the dimension of the integrals by one, i.e. instead of solving the integral for the whole domain only its contour needs to be considered.

Following the standard Bubnov-Galerkin procedure, the displacement field as well as the test function in each finite element e is approximated as

$$
\begin{aligned}
\mathbf{u}_{e} & =\mathbf{N}_{e} \mathbf{U}_{e}, \\
\mathbf{v}_{e} & =\mathbf{N}_{e} \mathbf{V}_{e} .
\end{aligned}
$$




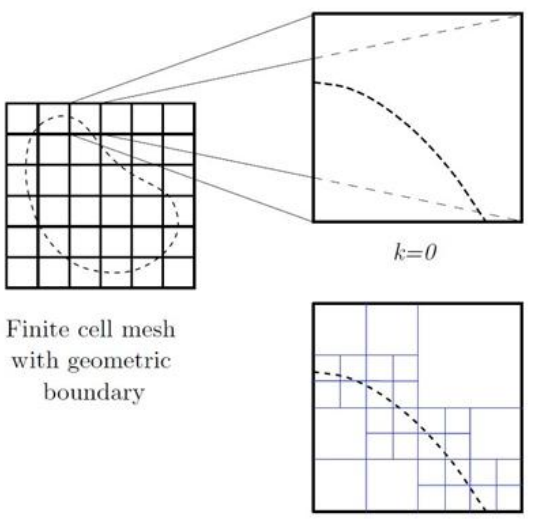

$k=3$

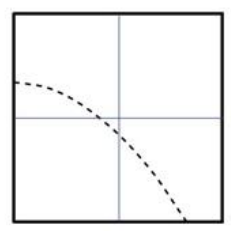

$k=1$

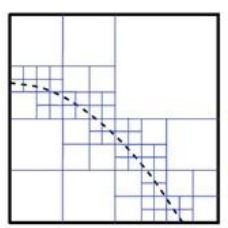

$k=4$

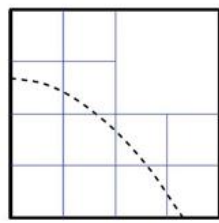

$k=2$

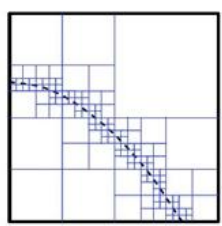

$k=5$

Figure 3. Adaptive subdivision of one element in integration cells

Here $\mathbf{N}_{e}$ contains the element shape functions, $\mathbf{U}_{e}$ represents the vector of unknowns, and $\mathbf{V}_{e}$ stands for the coefficients of the test functions for one single finite element, which in the context of FCM is usually named a finite cell. Inserting equations 2.10 and 2.11 into the weak form of equation (2.6) finally yields the well known linear system of equations

$$
\mathbf{K U}=\mathbf{F},
$$

where $\mathbf{K}$ denotes the global stiffness matrix and $\mathbf{F}$ represents the global load vector. The most important difference of the finite cell method to the standard finite element approach is the integration over the finite elements (finite cells), which are cut by the boundary (Figure 3).

For a more detailed insight into FCM we refer the reader to the comprehensive review article by Schillinger et al. 25] and the works by Düster et al. 15] and Parvizian et al. 16]. Here also several methods to include Dirichlet and Neumann types of boundary conditions are presented.

\section{Application of CT DAta in FCM simulation}

CT measurements result in a three-dimensional voxel data set containing all necessary information with respect to the microstructure. The voxel data can be further processed to obtain a boundary representation of the structure, e.g. via the surface tessellation language (STL) representation [26]. The determination of the material properties is based on the Hounsfield units (HU) of CT data. Both voxel data and STL data can be used as input data for simulation purposes.

The data from CT scans, given in STL format, can be processed by almost every CAD program. The file format can be either ASCII or binary. For processing STL data it is important that the triangular surface is closed and unique. Unfortunately, 

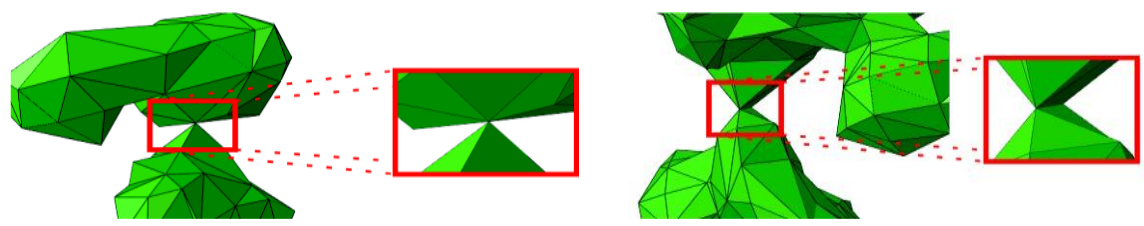

Figure 4. Problems of identifying closed STL surfaces [22]: left: two surfaces connected by a unit point; right; two surfaces connected by a unit line

the simplicity of STL format using unstructured triangular facets means some trouble shootings regarding the 3D finite element meshing process. The STL data often describe unclosed surfaces, or overlapping facets and incorrect normal directions. Additionally, sometimes two closed surface triangulations are connected to each other, as for instance by a line segment or by a point (see Figure 4).

The above mentioned problems complicate the unique identification of surface regions in an automated algorithm. Therefore a mesh repair or a remeshing procedure is absolutely essential for an FCM application. This is a highly complex issue and the topic of several publications 27, 28.

\section{INTEGRATION OF FCM into A COMMERCIAL FE PACKAGE}

Initially we developed and applied FCM for the analysis of ultrasonic waves propagating in heterogeneous materials. This development was part of an interdisciplinary research project, which was aimed at new wave based methods for structural health monitoring. The developed methods are focused on light-weight structures made from fiber and particle reinforced structures for airplanes or the rotor blades of wind power stations. Our FCM software development is a Matlab based in-house code, see [19, 29]. But, the FCM approach is also an interesting approach in other fields of applications, such as for the stress analysis of structures made from materials with a cellular microstructure. One important industrial application is the evaluation of aluminum die cast parts and their quality assurance by taking into account their inevitable porosity 21,22$]$. Today highly stressed parts are inline measured with CT in order to eliminate manufactured parts that do not meet the quality standards of the pore specification.

The FCM method cannot be well applied utilizing a university based in-house software which was developed for scientific reasons only. With regard to practical applications we are convinced that a robust implementation of the FCM methodology within a wide-spread and established software tool like Abaqus would create higher applicability to practical engineering problems. Therefore, we developed a software concept to couple the FCM with the commercial software package Abaqus, including open source software products for the pre- and post-processing tasks. The general workflow of our software concept is illustrated in Figure 5 


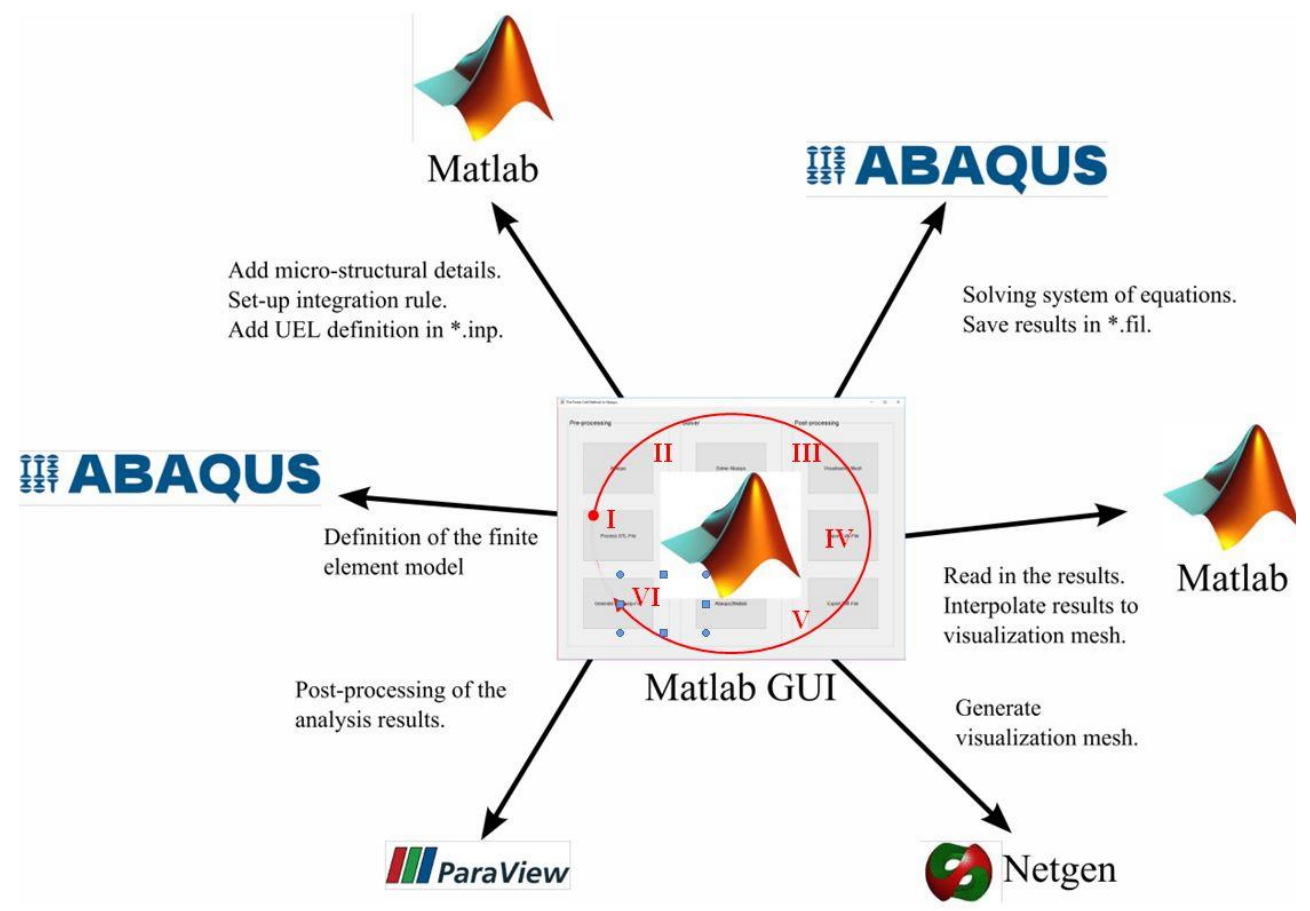

Figure 5. Workflow of the coupling of FCM with the FEM package Abaqus; the numbers I-VI denote the sequence of the application of the subprograms

For the coupling only free of access available software interfaces of Abaqus are used. To this end, we have defined a user subroutine with help of the Abaqus routine UEL that is able to incorporate the required functionality. In 3D the FCM can be applied if the FEM basis elements are hexahedral as well as tetrahedral finite elements [30]. Details concerning the required input data and the necessary pre- as well as postprocessing tools - although not directly related to Abaqus - are provided as well. Besides microstructural data from CT measurements, also virtual generated STL data can be applied in the design process of the constructions under investigation.

The initial model is set up in the pre-processing module of Abaqus. Here, the material properties and the element types are defined. In the next step an Abaqus input file is generated. This file is further processed in MATLAB and adjusted to incorporate the user defined element routine (UEL). At this stage the micro-structural details from CT measurements are added to the analysis and also the necessary details to perform the composed numerical integration are generated. During the solution of the governing equations these data are read in by the UEL. For the post-processing a geometry-conforming visualization mesh is created. This can be achieved using 
powerful mesh generators (e.g. Netgen [31]) or Abaqus itself. The analysis results are accordingly interpolated onto the new visualization nodes using the finite element shape functions of the coupled FEM-FCM model and saved in a vtk-file format. This format can be processed by ParaView, which offers all capabilities of commercial FE post-processing tools.

\section{Test EXAmple}

The developed coupled FEM-FCM concept based on Abaqus has been tested with help of several simple test examples, where as reference solutions overkill pure Abaqus solutions were used. The following test example demonstrates the capability of the developed coupling procedure 22].

In Figure 6 a cube with an edge length of $a=10 \mathrm{~m}$ is shown. A tensile load of $p=100 \mathrm{~N} / \mathrm{m}^{2}$ is applied normal to the positive $z$-direction. On the opposite surface the displacements in z-direction are constrained to zero. Additionally the displacements at two edges are also constrained to zero in $\mathrm{x}$ - and in y-direction, respectively. A Young's modulus of $70000 \mathrm{~N} / \mathrm{mm}^{2}$ and a Poisson ratio of 0.33 are used.
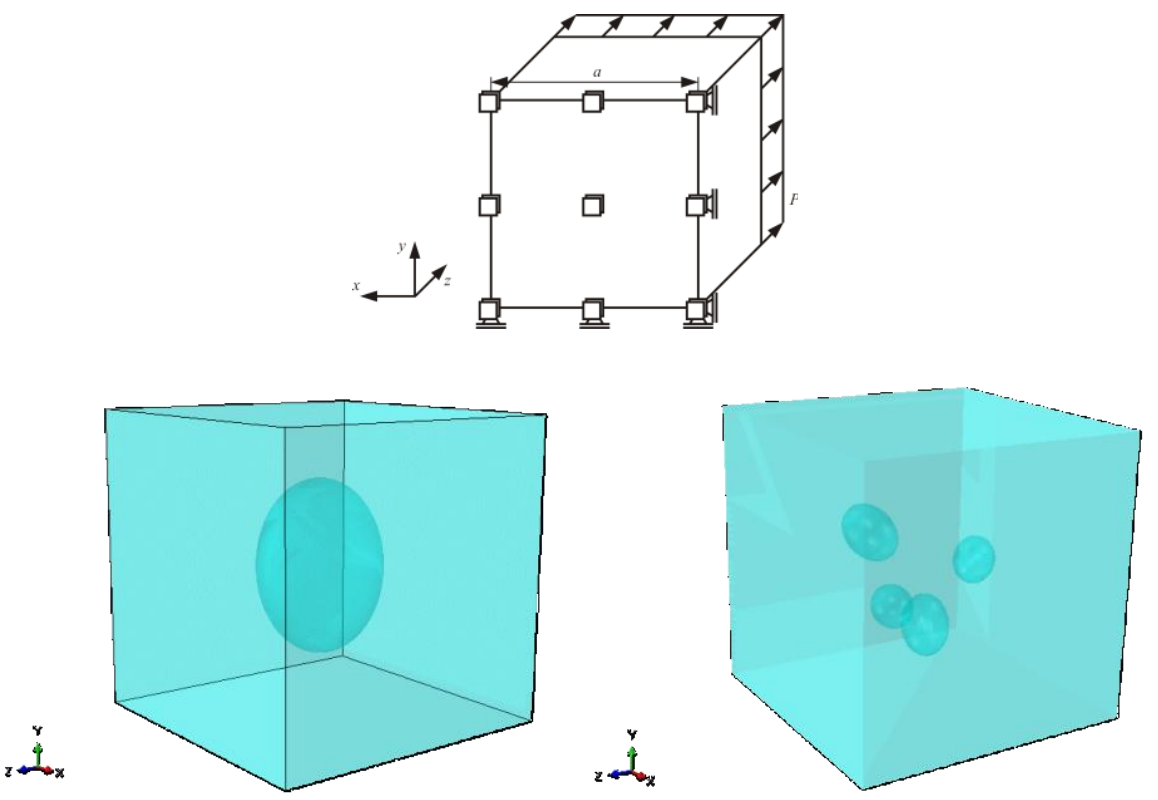

Figure 6. Cube with two types of inclusions: one centrally placed ellipsoid, and four randomly distributed ellipsoids

In the solid cube two versions of pores are embedded, a centrally embedded ellipsoidal pore in the first example and four randomly distributed embedded ellipsoidal pores in the second example, where the pores have different volumes and size (see Figure 6 . 
Cube with one central placed ellipsoid

The FE-FC model consists of $25 \times 25 \times 25$ hexahedral elements of second polynomial order (20 node hexahedrons). The cut elements are integrated with a subdivision level $k=3$ (for $k$ see Figure 3). The overkill reference Abaqus model consists of 180,325 second order tetrahedral elements (764,424 DOFs). In Figure 7 the von Mises stress results of the coupled FEM-FCM approach and the pure Abaqus solution are compared. The results are in a very good agreement, with an error of about $0.6 \%$.
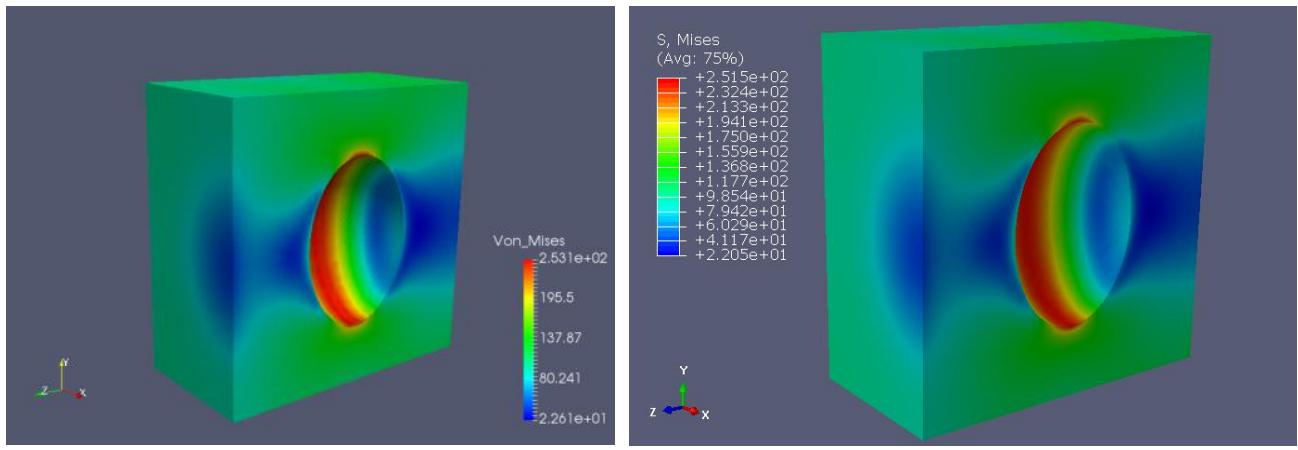

Figure 7. Von Mises stresses in a cube with a central ellipsoid; left: coupled FE-FC solution; right: overkill Abaqus reference solution

Cube with four randomly distributed ellipsoids

The FE-FC model consists again of $25 \times 25 \times 25$ hexahedral elements of second polynomial order (20 node hexahedrons). The cut elements are here integrated with a subdivision level $\mathrm{k}=4$ due to the smaller and more closely placed ellipsoids. The reference Abaqus model consists of 300,864 tetrahedral elements (1,263,081 DOFs). In Figure 7 the von Mises stress results of the coupled FEM-FCM approach and the pure overkill Abaqus solution are compared. The results are again in a good agreement, with a maximum error of $6 \%$.
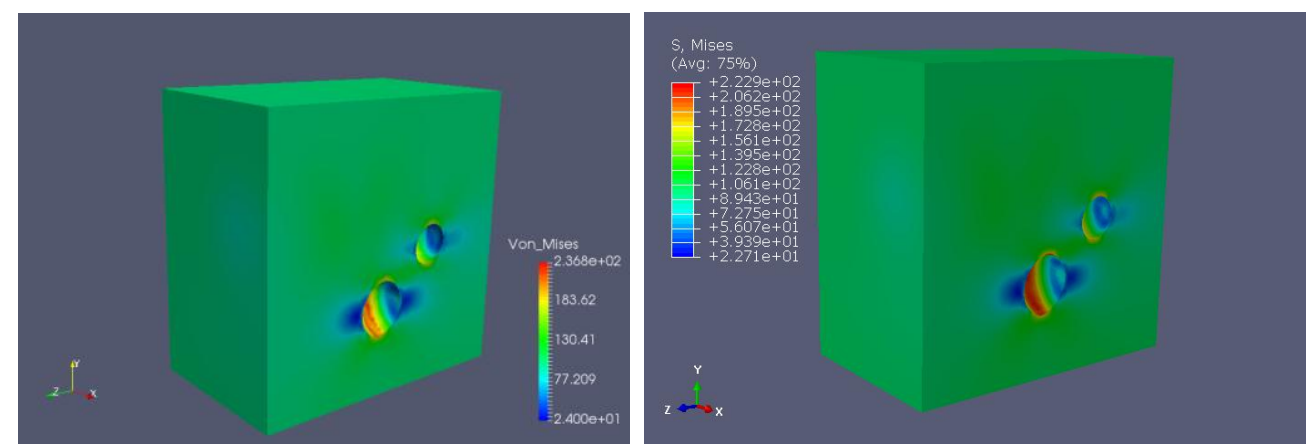

Figure 8. Von Mises stresses in a cube with a central ellipsoid; left: coupled FE-FC solution; right: overkill Abaqus reference solution 
Regarding the convergence of FCM we refer to [16, where the convergence properties of FCM are investigated in detail. It is shown that the convergence properties of the FCM can be directly derived from those of the associated finite element computation. In our case the FCM approach is coupled with the Abaqus software, where the accuracy can be increased with h-refinements only. In this case the convergence rate of the coupled FE-FC approach is algebraic and identical to the well known h-extension of the applied finite elements. It is important that the stiffness matrices of the cut finite elements are sufficiently accurately integrated, e.g. by an adaptive integration approach (see Figure3). For more details regarding the numerical integration we refer to $15,24,32$. If the mesh of the above given test examples is refined by increasing the number of hexahedral elements, the solution will converge to the Abaqus reference solution.

\section{Conclusion}

In the paper a concept for an implementation of the finite cell method (FCM) within the commercial software Abaqus is presented. The FCM can be efficiently applied for the stress and strain analysis of structures made from light-weight materials with a cellular or porous microstructure. The great advantage is inherent automated mesh generation with a quite regular hexahedron or tetrahedron mesh, which is not forced to match with the physical geometry of the structure. The real geometry is taken into account in the integration process of the element stiffness and load matrices of the cut finite elements (finite cells). The uncut finite elements are processed as in the standard FEM procedure. The cut elements are treated with an adaptive integration algorithm, as shown in Figure 3. The FCM provides a fast convergence rate if high order shape functions are used for the approximation of the displacement field in the finite elements. This is especially important if a very fine microstructure has to be taken into account with relative large finite elements/cells. In order to apply this methodology for the solution of engineering problems the FCM should be coupled with any standard commercial FEA software, such as Abaqus, Ansys, Nastran etc., which are powerful tools for solving complex engineering problems. In this paper the concept of a coupled FEM-FCM methodology has been presented and realized by using the Abaqus software. It is necessary to provide the microstructure in the form of a STL data file. The STL data can be derived by the CT measurements or can also be automatically generated. It is important that these STL data are unique and provide closed surfaces. This is a great problem if CT data in form of voxel data are used that are automatically transformed in STL data. Typically such data have to be repaired to be usable for the coupled FEM-FCM simulation. The applicability of the developed coupling method has been shown with academic test examples. The coupled FEM-FCM approach can also be used to solve industrial problems.

With the proposed methodology to couple FCM with a commercial finite element package like Abaqus, an important step has been taken towards a standardized analysis method for light-weight structures made from materials with a cellular microstructure. 


\section{REFERENCES}

1. O. C. Zienkiewicz and R. L. Taylor. The Finite Element Method - Solid Mechanics. 5th ed. Vol. 2. Butterworth-Heinemann, 2000.

2. K. J. Bathe. Finite Element Procedures. 2nd ed. Watertown, 2014.

3. B. Szabó and I. Babuška. Introduction to Finite Element Analysis: Formulation, Verification and Validation. John Wiley \& Sons, 2011.

4. R. Hill. "Continuum micromechanics of elastoplastic polycrystals." Journal of the Mechanics and Physics of Solids, 13(2), (1965), 89-101. DOI: 10.1016/ 0022-5096(65) 90023-2.

5. S. J. Hollister and N. Kikuehi. "A comparison of homogenization and standard mechanics analyses for periodic porous composites." Computational Mechanics, 10, (1992), 73 - 95. DOI: $10.1007 / \mathrm{BF} 00369853$

6. G. Lielens. "Micro-macro modeling of structured materials." PhD. Universite Catholique de Louvain, 1999. 141 pp. URL: https://d-nb.info/1005303312/ 34.

7. S. Kari. "Micromechanical modeling and numerical homogenization of fibre and particle reinforced composites." PhD. Düsseldorf: in Fortschritt-Berichte VDI., Reihe 18, Mechanik/Bruchmechanik; nr. 308: University of Magdeburg, Germany, 2007.

8. R. Rodríguez-Ramos, R. Guinovart-Díaz, J. Bravo-Castillero, F. J. Sabina, H. Berger, S. Kari, and U. Gabbert. "Variational bounds for anisotropic elastic multiphase composites with different shapes of inclusions." Archive of Applied Mechanics, 79, (2009), 695-708. DOI: 10.1007/s00419-008-0246-1.

9. A. Düster, H. G. Sehlhorst, and E. Rank. "Numerical homogenization of heterogeneous and cellular materials utilizing the finite cell method." Computational Mechanics, 50, (2012), 413-431. DOI: 10.1007/s00466-012-0681-2.

10. Chen Yang, L. Dong, B. Wang, Chen Yuli, Z. Qiu, and Z. Guo. "A substructurebased homogenization approach for systems with periodic microstructures of comparable sizes." Computational Mechanics, 50, (2017), pp. 97-104. DOI: 10. 1016/j.compstruct.2017.01.050.

11. F. Feyel. "A multilevel finite element method (FE2) to describe the response of highly non-linear structures using generalized continua." Computer Methods in Applied Mechanics and Engineering, 192(28-30), (2017), 3233-3244. DOI: 10.1016/S0045-7825(03)00348-7.

12. H. G. Sehlhorst. Numerical Homogenization Strategies for Cellular Materials with Applications in Structural Mechanics. Fortschritt-Berichte VDI: Reihe 18, Mechanik/Bruchmechanik. 2012. URL: https://books.google.hu/books?id= 5tg8MwEACAAJ.

13. J. A. Cottrell, T. J . R. Hughes, and Y. Bazilevs. "Isogeometric Analysis: Toward integration of CAD and FEA." Wiley, Hoboken, Sept. 2009. DOI: 10.1002/ 9780470749081.ch7.

14. J. Kastner and C. Heinzl. "X-ray computed tomography for non-destructive testing and materials characterization." Integrated Imaging and Vision Techniques 
for Industrial Inspection. Ed. by Liu Z., H. Ukida, P. Ramuhalli, and K. Niel. Springer, 2015, pp. 227-250. DOI: 10.1007/978-1-4471-6741-9_8.

15. A. Düster, J. Parvizian, Z. Yang, and E. Rank. "The finite cell method for threedimensional problems of solid mechanics." Computational Methods in Applied Mechanics and Engineering, 197(45), (2008), 3768-3782. DOI: $10.1016 / \mathrm{j}$. cma.2008.02.036.

16. J. Parvizian, A. Düster, and E. Rank. "The finite cell method for three-dimensional problems of solid mechanics." Computational Mechanics, 45, (2007), 121-133. DOI: $10.1007 / \mathrm{s} 00466-007-0173-y$.

17. M. Joulaian, S. Duczek, U. Gabbert, and A. Düster. "Finite and spectral cell method for wave propagation in heterogeneous materials." Computational Mechanics, 54, (2014), 661-675. DOI: 10.1007/s00466-014-1019-z.

18. C. Willberg, S. Duczek, J. M. Vivar Perez, D. Schmicker, and U. Gabbert. "Comparison of different higher order finite element schemes for the simulation of Lamb waves." Computer Methods in Applied Mechanics and Engineering, 54, (2014), pp. 241-244. DOI: 10.1016/j.cma.2012.06.011

19. S. Duczek. "Higher order finite elements and fictitious domain concept for wave propagation analysis." PhD. Düsseldorf: in Fortschritt-Berichte VDI., Reihe 20, Mechanik/Bruchmechanik; nr. 437: University of Magdeburg, Germany, 2014.

20. S. Duczek and U. Gabbert. "The Finite Cell Method: A higher order fictitious domain approach for wave propagation analysis in heterogeneous structures." Lamb-Wave Based Structural Health Monitoring in Polymer Composites, ed. by R. Lammering, U. Gabbert, M. Sinapius, T. Schuster, and P. Wierach. Springer, 2018, pp. 217-239. DOI: 10.1007/978-3-319-49715-0

21. S. Duczek, H. Berger, and U. Gabbert. "The Finite Pore Method: A new approach to evaluate gas pores in cast parts by combining computed tomography and the finite cell method." International Journal of Cast Metals Research, 28(4), (2015), pp. 221-228. DOI: 10.1179/1743133615Y.0000000003.

22. M. Würkner, S. Duczek, H. Berger, H. Köppe, and U. Gabbert. "A software platform for the analysis of die-cast parts with pores by the finite cell method," Analysis and Modelling of Advanced Structures and Smart Systems, ed. by H. Altenbach, E. Carrera, and G. Kulikov. Series: Advanced Structured Materials. Springer, Singapore, 2018, pp. 327-341. DOI: 10.1007/978-981-10-6895-9_14.

23. M. Ruess, D. Schillinger, Y. Bazilevs, V. Varduhn, and E. Rank. "Weakly enforced essential boundary conditions for NURB-embedded and trimmed NURBS geometries on the basis of the finite cell method." International Journal of Numerical Methods in Engineering, 95, (2013), pp. 811-846. DOI: 10.1002/ nme.4522,

24. S. Duczek and U. Gabbert. "Efficient integration method for fictitious domain approaches." Computational Mechanics, 56(4), (2015), pp. 725-738. DOI: 10 . 1007/s00466-015-1197-3.

25. D. Schillinger, Q. Cai, R. P. Mundani, and E. Rank. "A review of the finite cell method for nonlinear structural analysis of complex CAD and image based geometric models." Advanced Computing, Lecture Notes in Computational Science and Engineering, 93, (2013), pp. 1-23. DOI: 10.1007/978-3-642-38762-3_1 
26. E. Bechet, J. C. Cuilliere, and F. Trochu. "Generation of a finite element mesh from stereolithography (STL) files." Computer-Aided Design, 34(1), (2002), pp. 117. DOI: $10.1016 / \mathrm{S} 0010-4485(00) 00146-9$.

27. F. Liu, H. Zhou, and D. Li. "Repair of STL errors." International Journal of Production Research, 47(1), (2009), pp. 1-23. DOI: 10.1080/00207540701424539.

28. M. Attene. "Direct repair of self-intersecting meshes." Graphical Models, 76(6), (2014), 658 - 668. DOI: $10.1016 / \mathrm{j} . \operatorname{gmod} .2014 .09 .002$.

29. R. Lammering, U. Gabbert, M. Sinapius, T. Schuster, and P. Wierach, eds. Lamb-Wave Based Structural Health Monitoring in Polymer Composites. Springer International Publishing, 2018. URL: 10.1007/978-3-319-49715-0.

30. S. Duczek, F. Duvigneau, and U. Gabbert. "The finite cell method for tetrahedral meshes." Finite Elements in Analysis and Design, 121, (2016), 18-32. DOI: 10. 1016/j.finel.2016.07.004.

31. J. Schöberl. "NETGEN: An advancing front 2D/3D-mesh generator based on abstract rules." Computing and Visualization in Science, 1, (1997), 41-52. DOI: $10.1007 / \mathrm{s} 007910050004$

32. Z. Yang, M. Ruess, S. Kollmannsberger, A. Düster, and E. Rank. "An efficient integration technique for the voxel-based finite cell method." Journal for $\mathrm{Nu}$ merical Methods in Engineering, 91(5), (2012), 457-471. 